\title{
Para além da barreira dos números: desigualdades raciais e saúde
}

\author{
Beyond the numbers barrier: \\ racial inequalities and health
}

\author{
1 Programa de Combate \\ ao Racismo Institucional, \\ Ministério Britânico \\ para o Desenvolvimento \\ Internacional - Escritório \\ Brasil, Brasília, Brasil. \\ Correspondência \\ F. Lopes \\ Programa de Combate \\ ao Racismo Institucional, \\ Ministério Britânico \\ para o Desenvolvimento \\ Internacional - Escritório \\ Brasil. Edifício Centro \\ Empresarial VARIG, SCN \\ Quadra 4, Bloco B, Torre \\ Oeste, Sala 202, Brasília, DF \\ 70714-900, Brasil. \\ f-lopes@dfid.gov.uk \\ lopesf@usp.br
}

\begin{abstract}
The point of departure for this article was the concept of health as a set of comprehensive and collective living conditions, influenced by the political, socioeconomic, cultural, and environmental context. The work thus shows that studies on health inequalities, disparities, or iniquities should extend beyond statistical data, since racism is not always explicit and measurable in social interactions. It is necessary to analyze the various life experiences of blacks and non-blacks in a given social condition, considering gender, age, place of residence, schooling, family origin, occupation, income, sexual orientation, religious affiliation, capacities and incapacities, social network, and possibilities for accessing social goods and services. Finally, the article lists guidelines that can assist in the major challenge of drafting public policies to combat and eradicate the immense inequalities between whites and blacks.
\end{abstract}

Prejudice; Health Inequity; Equity
Fernanda Lopes 1

Ao reconhecer a saúde como o conjunto de condições integrais e coletivas de existência, influenciado pelo contexto político, sócio-econômico, cultural e ambiental, a comunidade acadêmica reitera a necessidade e importância dos estudos sobre o impacto das desigualdades sociais nas condições de saúde, seja do ponto de vista individual ou coletivo.

No entanto, excetuando-se poucas experiências, alguns fatores constitutivos dos processos de organização e hierarquização social são, continuamente, desconsiderados em estudos e pesquisas sobre desigualdades, disparidades ou iniqüidades em saúde. O sexismo, o racismo, o etnocentrismo e outros integram a lista de fatores descartados ou descartáveis.

No Brasil e em outras sociedades modernas, as hierarquias sociais são justificadas e racionalizadas de diferentes modos, e todos eles, sem exceção, apelam à ordem "natural" de sua existência e apresentam-se como um traço constitutivo das relações sociais. Segundo Guimarães 1, o sistema de hierarquização brasileiro, e mesmo o latino-americano, interliga cor da pele, classe (ocupação e renda) e status social (sexo, origem familiar, educação formal, condições de moradia e habitação), sem, contudo, deixar de ser sustentado pela dicotomia racial branco versus preto que alicerçou a ordem escravocrata por três séculos e que, na atualidade, resiste à urbanização, à industrialização, às mudanças de sistema e regimes políticos. 
Ao demarcar os espaços sociais com base na aparência e na cor da pele, as sociedades latino-americanas, e a brasileira em particular, resgatam a natureza sócio-histórica do conceito de raça e capturam seu potencial de identificar diferentes sentidos e significados no acesso ao poder, no acesso ao repertório de direitos efetivamente disponíveis e ao conjunto de recursos socialmente desejáveis. Nesse sentido, o racismo enquanto fenômeno ideológico submete todos sem distinção; revitaliza e mantém sua dinâmica com a evolução da sociedade, das conjunturas históricas e dos interesses dos grupos dominantes.

Os perversos efeitos dessa programação social sobre a população negra são inúmeros (diversos e assimétricos nas várias fases do ciclo de vida) e podem ser evidenciados, direta ou indiretamente, a partir da análise de alguns aspectos das relações interpessoais e das relações que o grupo estabelece com as instituições; da análise de sua situação sócio-econômica, condições de vida e de desenvolvimento humano, participação no mercado de trabalho, acesso aos bens e equipamentos sociais e de sua morbimortalidade, como observam Cavalleiro 2, Martins 3, Hasenbalg 4, Cunha 5, Jaccoud \& Beghin 6, Paixão 7, Batista et al. 8, Martins \& Tanaka 9 , Wood \& Carvalho 10 , dentre outros.

\section{Algumas evidências das desigualdades sócio-raciais no Brasil}

\section{Concentração de riqueza e pobreza}

No Brasil, o grau de pobreza é mais elevado do que o encontrado em outros países com renda per capita similar. Embora cerca de $64,0 \%$ dos países tenham renda inferior à brasileira, aqui o grau de desigualdades é um dos mais elevados do mundo 11 .

Ao estudar os ricos brasileiros, Medeiros 12 afirma que a dimensão espaço-regional é essencial para a compreensão e redução das desigualdades sociais. Segundo o autor, são expressivas as diferenças nos níveis de renda per capita entre regiões, sendo maior a concentração de rendimentos nas áreas mais pobres. Em termos de renda média, nível e crescimento de renda, as regiões Sul e Sudeste são evidentemente mais ricas, Norte e Nordeste mais pobres 12,13.

Dados de 2001 do Instituto de Pesquisa Econômica Aplicada (IPEA), destacados por Sant'Anna 14, comprovam a estrutura racial de distribuição de riqueza: no Nordeste, a razão entre a renda apropriada pelos $20,0 \%$ mais ricos e aquela apropriada pelos $20,0 \%$ mais po- bres foi de 34,7 para a população branca e 19,6 para a negra; no Sudeste foi de 21,0 e 15,8; e no Sul, 18,8 e 15,9, respectivamente. Observe-se que, em 2000, os negros representavam $65,7 \%$ da população nordestina. Em relação à população brasileira, o grupo correspondia a 45,0\% e era responsável por apenas $27,0 \%$ do rendimento domiciliar per capita total do país, enquanto os não-negros, 54,3\% da população, detinham $73,0 \%$ do total desse rendimento 15 .

Em 1999, a média de renda per capita nos domicílios com chefia negra correspondia a $42,0 \%$ dos valores observados naqueles com chefia branca 16 . Em 2001, vivia-se com R\$ 205,40 nos domicílios negros e pouco mais que o dobro nos domicílios brancos. Embora a média de renda per capita domiciliar persista baixa para o conjunto da população ( $R \$ 482,10)$, detecta-se queda na proporção de pobres: em 1992, estes eram 40,7\%; em 2001 passaram a $33,6 \% 6$. Ao desagregar os dados por raça/cor, também foi possível observar redução, contudo, a proporção de negros pobres manteve-se duas vezes à observada para brancos pobres: $55,3 \%$ versus $28,9 \%$ em 1992 e $46,8 \%$ versus 22,4\% em 20016.

É nítida a dimensão espaço-regional na concentração de riqueza e na distribuição dos pobres e inegável sua relação com raça/cor e gênero - as mulheres negras encontram-se nos mais baixos patamares de renda -, reiterando a literatura nacional e internacional 17,18,19,20,21.

Embora a sociedade brasileira não conviva com o ódio racial, com a segregação legal ou explícita, a atribuição de um significado social negativo a determinados padrões fenotípicos de diversidades justifica o tratamento desigual, impõe e intercala barreiras que impedem ou dificultam a mobilidade social negra 22 . A sobre-representação do negro na pobreza, com especial destaque para a situação da mulher negra, restringe suas possibilidades de consumo, de acesso aos bens sociais potencialmente disponíveis e, por conseqüência, suas liberdades individuais. Como afirmou Santos 23, aos pobres é oferecida uma cidadania abstrata, que não cabe em qualquer tempo e lugar e que, na maioria das vezes, não pode ser sequer reclamada.

\section{Condições de moradia e habitação}

Nos anos de 1990 melhoraram as condições habitacionais de toda a população. Entretanto, em 2001, a proporção de negros que vivia em aglomerados subnormais (favelas, mocambos, palafitas e similares) ainda era quase o dobro da proporção de brancos. Todavia a definição 
do Instituto Brasileiro de Geografia e Estatística (IBGE) para favela deva ser revista e alterada 24 , não deve ser ignorado o fato de que em 2001, três quartos das pessoas que viviam em favelas eram negras (autodeclaradas pretas ou pardas) 6 .

Com o passar dos anos, as disparidades entre negros e brancos, quanto às condições de moradia e habitação nos domicílios particulares permanentes urbanos, também foram reduzidas em diversos aspectos: condições físicas e de ocupação do espaço; saneamento; abastecimento público de água; coleta de lixo; energia elétrica; e presença de banheiro de uso exclusivo. Contudo, a sobre-representação dos negros entre os destituídos desses bens potencialmente disponíveis manteve-se em patamares elevados, independentemente da região geográfica considerada, com exceção do acesso à energia elétrica.

Do ponto de vista da saúde, as condições desfavoráveis de moradia e habitação propiciam a disseminação de doenças respiratórias, infecciosas e parasitárias 25 , e potencializam as situações de violência sexual, física e psicológica 26. Para além do incremento das vulnerabilidades às patologias ou aos agravos citados, o estresse cotidiano e a insatisfação com o meio onde vivem (espaço físico e simbólico) também alteram a qualidade de vida auto-atribuída.

\section{Acesso à educação}

As políticas públicas projetadas para diminuir os índices de analfabetismo no Brasil têm resultados incontestáveis e uma progressão mais promissora para as mulheres que para os homens: em 1940, apenas metade da população masculina e $36,0 \%$ da feminina com dez anos e mais era alfabetizada; após quarenta anos, essa taxa era de $76,0 \%$ para homens e $74,0 \%$ para mulheres; e, em 2000 , passou para $87,8 \%$ e $88,0 \%$, respectivamente 27 . O caráter promissor das iniciativas universalistas na área da educação passa a ser questionado quando são comparados os perfis de escolaridade de brancos e negros, especialmente nas faixas etárias de 15 a 24 anos, de 45 anos e mais, e nas regiões mais desenvolvidas. Em 1992, a taxa de analfabetismo para a população de 15 anos e mais era de $25,7 \%$ para os negros e $10,6 \%$ para os brancos; uma década depois, $18,2 \%$ dos negros continuavam analfabetos contra $7,7 \%$ dos brancos. De acordo com os dados censitários de 2000, a população branca estudava 6,9 anos e a negra, 4,7 anos. No Sudeste, onde se encontra a maior média para a população geral ( 6,7 anos), os negros estudavam, em média, 2,1 anos menos que os brancos 6 .
Em qualquer sociedade, o sistema educacional pode se constituir em poderoso agente de inclusão social e de promoção da igualdade. No Brasil, porém, a negligência do ensino diante das iniqüidades geradas por séculos de escravidão potencializa a manutenção e ampliação das disparidades. No início do século 21, mais da metade da população negra adulta (25 anos e mais) tinha menos de quatro anos de estudo (analfabetos funcionais); 82,0\% (contra menos de dois terços dos brancos) não haviam completado o primeiro grau (oito anos de estudo); 90,0\% (contra três quartos dos brancos) não chegaram a terminar o ensino médio (11 anos de estudo); e apenas dois entre cem negros adultos concluíram quatro anos de ensino superior (contra cerca de dez brancos) 6 .

É preocupante a extensão e persistência da exclusão da população negra do nível superior de ensino, dado que essa situação significa um virtual alijamento das ocupações de maior prestígio e remuneração; das proposições de comando e deliberação; das camadas dirigentes dos setores público e privado; das atividades culturais e científicas que demandam educação formal.

Embora a escolaridade não seja a variável com maior poder explicativo no desencadeamento dos processos de adoecimento ela figura como elemento de suma importância ao se tratar do acesso aos serviços, da comunicação com o profissional de saúde - em especial, com o médico -, da conseqüente efetividade na prevenção, tratamento e cura de doenças, bem como no que se refere aos processos de ressignificação, por parte da população, das noções de saúde e doença.

\section{Emprego e renda}

Em 1999, o índice de rendimento médio na ocupação principal de negros correspondeu a $48,0 \%$ do índice observado para a população branca 16,28. Num estudo de 2004, Martins 3, ao analisar os dados sobre a renda média da ocupação principal (padronizada para quarenta horas semanais), afirma que, todavia haja pequenas variações regionais em torno da média nacional, as disparidades de renda entre negros e brancos está presente em todas as regiões do país, independentemente do nível de desenvolvimento, das condições específicas do mercado de trabalho (ainda que seja atribuído aos dois grupos o mesmo perfil educacional e sejam mantidos os diferenciais de remuneração observados para cada faixa de escolaridade). Conquanto a educação formal catalise a mobilidade social, as possibilidades de ascen- 
são dos brancos, obtidas com o aumento do nível de escolaridade, são potencializadas com auxílio de sua rede social; para os negros, no entanto, o leque de oportunidades apresentase mais restrito, uma vez que as novas gerações (geralmente mais escolarizadas) ainda não estabeleceram uma rede social que lhes ofereça o suporte necessário para mudança de status.

Segundo Soares 29 , se não houvesse discriminação racial e de gênero, as mulheres negras ganhariam, em média, cerca de $60,0 \%$ mais; as brancas receberiam em torno de $40,0 \%$ e os homens negros, entre $10,0 \%$ e $25,0 \%$ mais (dependendo do lugar ocupado na distribuição de renda). As disparidades de renda observadas entre os grupos de raça/cor e sexo não derivam da heterogeneidade da distribuição racial da população ou ainda da heterogeneidade dos níveis de educação formal no território brasileiro, mas sim do próprio mercado de trabalho, que gera segmentação ocupacional e discriminação salarial baseadas em raça/cor e sexo 3,29,30,31.

\section{Outras marcas visíveis e mensuráveis}

\section{Desesperança de vida ao nascer}

No Brasil, a esperança de vida ao nascer, em 2000 , era de 70,4 anos. A dinâmica de gênero imputada ao indicador de longevidade, diferentemente do comportamento apresentado para os demais indicadores ou demais variáveis, ocorre em detrimento dos homens, com as mulheres vivendo, em média, 7,45 anos a mais que eles. Ao serem considerados os grupos de raça/cor, tal esperança era maior para os amarelos (75,75 anos), seguida daquela apresentada para os brancos (73,99 anos). Diferenças relevantes também foram observadas entre os demais grupos: para os pardos foi estimada uma expectativa de vida de 67,87 anos; para os pretos, de 67,64; e, para os indígenas, de 66,57 anos. Quando comparados aos brancos, os pretos apresentaram uma expectativa inferior de 6,35 anos e os pardos de 5,96 (no seu conjunto, a expectativa de vida dos negros foi 6,12 anos menor que a dos brancos). Cabe assinalar que, ainda em 2000, a esperança de vida de povos indígenas ao nascer era 7,42 anos inferior à dos brancos, 1,3 ano inferior à dos negros e 9,18 inferior à dos amarelos 32. As diferenças com base na pertença étnico-racial foram mantidas no interior dos grupos de sexo, com mulheres e homens brancos gozando de maior expectativa de vida quando comparados às mulheres e homens negros. Os dados apresentados por Paixão e colaboradores, inéditos até o presente momento, reiteram as evidências descritas anteriormente no Atlas de Desenvolvimento Humano 33.

\section{Causas externas de morte:} uma perda superlativa de vidas negras

Desde os anos de 1980, as causas externas representam a segunda causa de morte no Brasil, fato que tem atraído a atenção de inúmeros pesquisadores interessados no assunto; e também, do ponto de vista das políticas públicas, promovido crescente demanda dos serviços de saúde.

No triênio 1998/2000, um quarto dos óbitos declarados de homens negros foi atribuído às causas externas contra $16,0 \%$ para os brancos. Quase metade das mortes de negros foi oriunda de homicídios; para os brancos, a proporção foi de $34,4 \%$. Também foram mais freqüentes entre negros os óbitos determinados por ataque com arma de fogo (32,0\% versus $21,9 \%) 32$.

Ainda que a violência figure entre os principais problemas de saúde pública da atualidade, poucos estudiosos têm se dedicado a verificar as interseções estabelecidas entre o evento (morte, traumas, lesões, incapacidades) e local de moradia, gênero, faixa etária, educação formal, condições gerais de vida, ocupação, uso ou tráfico de drogas, orientação sexual, raça ou etnia. Nesse sentido, a ampliação do leque de pesquisas sobre as causas externas de morbimortalidade e a incorporação de novas perspectivas de análise e interpretação dos dados serão instrumentos úteis para a atuação no campo das políticas públicas, tanto do ponto de vista administrativo (planejamento de serviços e alocação de recursos), da avaliação e acompanhamento da qualidade e efetividade da assistência médica prestada, como da educação permanente (envolvimento dos profissionais que prestam atendimento direto às vítimas na discussão sobre as várias dimensões do tema).

\section{Considerações sobre universalidade, eqüidade e produção de conhecimento científico}

Ao instituir a saúde como direito de todos(as) e dever do Estado (Artigo 196 da Constituição Federal Brasileira), o Estado brasileiro assume a responsabilidade de garantir acesso universal e igualitário às ações e serviços de saúde, de modo a contemplar, da melhor forma possível, as necessidades e demandas da população.

Embora seja um avanço inegável como direito do cidadão, não o é quando trata do dever 
do Estado, pois este não tem assegurado a todos a mesma qualidade de atenção ou perfil de saúde, seja do ponto de vista regional e/ou étnico-racial 34. Mulheres e homens; populações indígenas, negros e brancos ocupam lugares desiguais nas redes sociais e trazem consigo experiências também desiguais de nascer, viver, adoecer e morrer. Diante de tal constatação, é essencial que, no campo das políticas públicas ou institucionais, o processo de definição de diretrizes e metas não restrinja o conceito de igualdade ao seu aspecto formal e que seja considerado o impacto de preposições e ações sobre grupos populacionais específicos.

O combate e a erradicação das desigualdades torna-se um grande desafio no campo das políticas públicas, especialmente quando tais desigualdades são oriundas da expressão indireta de discriminação. Poder público e sociedade civil precisam ser imparciais no enfrentamento de práticas, procedimentos ou leis que, explicitamente, desfavorecem pessoas ou grupos característicos e devem ter em vista que, para eliminar as formas indiretas de discriminação, é necessário: (1) mensurar e interpretar de forma correta a magnitude e a evolução das disparidades entre os grupos hegemônicos e não-hegemônicos; (2) revisar as práticas institucionais, de modo que as novas ações, programas e políticas sejam orientadas pela noção de eqüidade; (3) assumir o compromisso e a responsabilidade de oferecer tratamento diferente àqueles que estão inseridos de forma desi- gual, contemplando assim suas necessidades e promovendo ou efetivando o direito à igualdade de fato.

Do ponto de vista da pesquisa acadêmica, é preciso considerar que o racismo nem sempre se faz presente, de forma explícita e mensurável, nas interações. Desse modo, não basta que os estudiosos apresentem aos seus pares análises das diferenças numéricas com significância estatística. Seus estudos devem considerar o conjunto de fatores históricos, sócio-políticos, econômicos e culturais que contribuem para existência, manutenção ou ampliação dos diferenciais no interior dos grupos ou intergrupos. É preciso analisar as diversas experiências vivenciadas por negros e não-negros numa dada condição social, considerando sexo, idade, região de moradia, educação formal, origem familiar, ocupação, renda, orientação sexual, denominação religiosa, capacidades e incapacidades, rede social e comunitária, possibilidades de acesso aos serviços e aos bens sociais. Nesse sentido, a ausência de relevância estatística na distribuição e comportamento das variáveis por raça/cor não isenta o pesquisador da responsabilidade de reiterar tanto a natureza perversa do racismo, como sua capacidade e aptidão em criar e/ou perpetuar diferenciais nas condições gerais de vida nos grupos e intergrupos. É de sua responsabilidade indicar brechas a serem exploradas por outros estudos que adotem abordagens qualitativas ou quantiqualitativas.

\section{Resumo}

Este artigo parte do conceito de saúde como o conjunto de condições integrais e coletivas de existência, influenciado pelo contexto político, sócio-econômico, cultural e ambiental. Desse modo, mostra que os estudos sobre as desigualdades, disparidades ou iniqüidades em saúde devem ir muito além da comparação de dados estatísticos, uma vez que o racismo nem sempre se apresenta de forma explícita e mensurável nas interações sociais. É preciso analisar as diversas experiências vivenciadas por negros e não-negros numa dada condição social, considerando sexo, idade, região de moradia, educação, origem familiar, ocupação, renda, orientação sexual, filiação religiosa, suas capacidades e incapacidades, sua rede social e suas possibilidades de acesso aos serviços e bens sociais. Por fim, o artigo enumera diretrizes que possam colaborar com o grande desafio de formulação de políticas públicas que combatam e erradiquem as imensas desigualdades entre brancos e negros.

Preconceito; Iniqüidade na Saúde; Eqüidade 


\section{Referências}

1. Guimarães ASA. Raça, racismo e grupos de cor no Brasil. Estud Afro-Asiát 1995; 27:45-63.

2. Cavalleiro E. Racismo e anti-racismo na educação: repensando nossa escola. São Paulo: Summus; 2001.

3. Martins RB. Desigualdades raciais e políticas de inclusão racial: um sumário da experiência brasileira recente. Santiago do Chile: Comisión Económica para América Latina y el Caribe; 2004. (Serie Políticas Sociales 82). http://www.eclac.cl/ publicaciones/DesarrolloSocial/2/LCL2082PP/S erie82_P.pdf (acessado em 10/Set/2004).

4. Hasenbalg C. Entre o mito e os fatos: racismo e relações raciais no Brasil. In: Maio MC, Santos RV, organizadores. Raça, ciência e sociedade. Rio de Janeiro: Fundação Oswaldo Cruz/Centro Cultural Banco do Brasil; 1996. p. 235-49.

5. Cunha EMGP. Infant mortality and race: the differences of inequality. In: Hogan DJ, editor. Population change in Brazil: contemporary perspectives. Campinas: Núcleo de Estudos da População, Universidade Estadual de Campinas; 2001. p. 333-6.

6. Jaccoud LB, Beghin N. Desigualdades raciais no Brasil: um balanço da intervenção governamental. Brasília: Instituto de Pesquisa Econômica Aplicada; 2002.

7. Paixão M. Desenvolvimento humano e relações raciais. Rio de Janeiro: DP\&A; 2003.

8. Batista LE, Escuder MML, Pereira JCR. A cor da morte: causas de óbito segundo características de raça no Estado de São Paulo, 1999 a 2001. Rev Saúde Pública 2004; 38:630-6.

9. Martins AL, Tanaka ACD. Mulheres negras e mortalidade no Estado do Paraná, Brasil, de 1993 a 1998. Rev Bras Crescimento Desenvolv Hum 2000; 10:27-38.

10. Wood CH, Carvalho JAM. Categorias do censo e classificação subjetiva de cor no Brasil. Revista Brasileira de Estudos Populacionais 1994; 11:315.

11. Barros RP, Henriques R, Mendonça R. Desigualdade e pobreza no Brasil: retrato de uma estabilidade inaceitável. Rev Bras Ciênc Soc 2000; 15: 127-41.

12. Medeiros M. A geografia dos ricos no Brasil. Brasília: Instituto de Pesquisa Econômica Aplicada; 2004. (Texto para Discussão 1029). http:// www.ipea.gov.br/pub/td/2004/td_1029.pdf (acessado em 10/Set/2004).

13. Azzoni C, Menezes Filho N, Menezes T, Silveira Neto R. Geografia e convergência da renda entre os estados brasileiros. In: Henriques R, organizador. Desigualdade e pobreza no Brasil. Brasília: Instituto de Pesquisa Econômica Aplicada; 2000. p. 299-343.

14. Sant'Anna W. Dossiê assimetrias raciais no Brasil: alerta para a elaboração de políticas. Belo Horizonte: Rede Feminista de Saúde/Rede Nacional Feminista de Saúde, Direitos Sexuais e Direitos Reprodutivos; 2003. http://www.redesaude.gov. br (acessado em 10/Set/2004).

15. Kilsztajn S, Carmo MSN, Sugahara GTL, Lopes ES. Vítimas da cor: homicídios na região metropoli- tana de São Paulo, 2000. In: Congresso Brasileiro de Epidemiologia [CD-ROM]. Recife: ABRASCO; 2004.

16. Martins RB. Desigualdades raciais no Brasil. http://www.ipea.gov.br/pub/desigualdades_raciais.ppt (acessado em 15/Dez/2001)

17. Williams D. Race and health: basic questions, emerging directions. Ann Epidemiol 1997; 7:32233.

18. Krieger N, Rowley DL, Herman AA, Avery B, Phillips MT. Racism, sexism and social class: implications for studies of health, disease and wellbeing. Am J Prev Med 1993; 9 (2 Suppl):82-122.

19. Crenshaw K. Documento para o encontro de especialistas em aspectos da discriminação racial relativos ao gênero. Estudos Feministas 2002; 1: 171-88.

20. Beddola LG. Studying inequality: race, class, gender and sexuality. In: Consulado Geral dos Estados Unidos da América no Rio de Janeiro, organizador. A intersecção das desigualdades de raça e gênero: implicações para as políticas públicas e os direitos humanos. Rio de Janeiro: Instituto Brasileiro de Administração Municipal/Fundo de Desenvolvimento das Nações Unidas para a Mulher/Consulado Geral dos Estados Unidos da América no Rio de Janeiro; 2004. p. 1-22.

21. Bairros L. Lembrando Lélia Gonzalez. In: Consulado Geral dos Estados Unidos da América no Rio de Janeiro, organizador. A intersecção das desigualdades de raça e gênero: implicações para as políticas públicas e os direitos humanos. Rio de Janeiro: Instituto Brasileiro de Administração Municipal/Fundo de Desenvolvimento das Nações Unidas para a Mulher/Consulado Geral dos Estados Unidos da América no Rio de Janeiro; 2004. p. 31-46.

22. Silva NV. Uma nota sobre "raça social" no Brasil. Estud Afro-Asiát 1994; 26:67-80.

23. Santos M. Por uma outra globalização: do pensamento único à consciência universal. 4ạ Ed. Rio de Janeiro: Record; 2000.

24. Petreicelli E, Valladares 1 . A desigualdade entre os pobres - favela, favelas. In: Henriques R, organizador. Desigualdade e pobreza no Brasil. Brasília: Instituto de Pesquisa Econômica Aplicada; 2000. p. 459-85.

25. Rosemberg F, Pinto RP. Saneamento básico e raça. Rev Bras Crescimento Desenvolv Hum 1995; 5:2338.

26. Krieger J, Higgins D. Housing and health: time again for public health action. Am J Public Health 2002; 92:758-68.

27. Beltrão KI. Alfabetização por sexo e raça no Brasil: um modelo linear generalizado para explicar a evolução no período 1940-2000. Brasília: Instituto de Pesquisa Econômica Aplicada; 2004. (Texto para Discussão 1003). http://www.ipea.gov. $\mathrm{br} / \mathrm{pub} / \mathrm{td} / 2004 / \mathrm{td} \_1003 . p d f$ (acessado em 10/ Set/2004).

28. Instituto Sindical Interamericano pela Igualdade Racial. Mapa da população negra no mercado de trabalho: regiões metropolitanas de São Paulo, Salvador, Recife, Belo Horizonte, Porto Alegre e 
Distrito Federal. São Paulo: American Federation of Labor and Congress of Industrial Organizations; 1999.

29. Soares S. Discriminação de gênero e raça no mercado de trabalho. Boletim de Mercado de Trabalho - Conjuntura e Análise 2000; 13:45-51.

30. Ramos LR, Vieira ML. Determinantes da desigualdade de rendimentos no Brasil, nos anos 90: discriminação, segmentação e heterogeneidade dos trabalhadores. In: Henriques R, organizador. Desigualdade e pobreza no Brasil. Brasília: Instituto de Pesquisa Econômica Aplicada; 2000. p. 159-76.

31. Ferranti D, Perry GE, Ferreira FHG, Walton M, Coady D, Cunningham W, et al., editors. Groupbased inequalities: the roles of race, ethnicity and gender. In: Advance Conference Edition of Inequality in Latin America and the Caribbean breaking with History? Washington DC: The International Bank for Reconstruction and Development/World Bank; 2003. p. 81-126.
32. Paixão MJP, Carvano LMF, Oliveira, JC, Ervatti, LR. Diferenciais de esperança de vida e de anos de vida perdidos segundo os grupos de raça/cor e sexo no Brasil e grandes regiões. In: Lopes F, organizador. Saúde da população negra no Brasil: contribuições para a promoção da eqüidade. Brasília: Fundação Nacional de Saúde, Ministério da Saúde; 2004. (mimeo.).

33. Programa das Nações Unidas para Desenvolvimento. Atlas de desenvolvimento humano no Brasil. http://www.pnud.org.br/atlas (acessado em 10/Set/2004).

34. Ministério da Saúde. Caderno de Textos Básicos Seminário Nacional de Saúde da População Negra 2004 ago 18-20; Brasília: Ministério da Saúde; 2004. http://www.dtr2002.saude.gov.br/saudenegra/ (acessado em 10/Set/2004).

Recebido em 06/Jan/2005

Versão final reapresentada em 22/Fev/2005

Aprovado em 03/Mai/2005 